\title{
Waiting List
}

National Cancer Institute

\section{Source}

National Cancer Institute. Waiting List. NCI Thesaurus. Code C154226.

A list of people waiting for inclusion in some activity, vacancy, or service. 